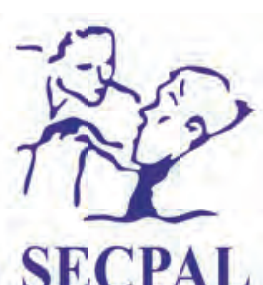

Medicina Paliativa

www. medicinapaliativa.es

EDITORIAL

\title{
Planificación anticipada de decisiones: un instrumento clínico esencial para la calidad asistencial
}

La escritora y diplomática estadounidense Eleanor Roosevelt, considerada una de las líderes más influyentes del siglo xx, fue la principal impulsora de la Declaración Universal de los Derechos Humanos de 1948, y una de las mayores luchadoras por la igualdad de las mujeres. Sin embargo, la atención médica que recibió en la última etapa de su vida, condicionada por una anemia aplásica, le originó un tremendo sufrimiento y fue una flagrante violación de todos los principios bioéticos que estaban comenzando a enunciarse ${ }^{1}$. No es casualidad que, en ese contexto, fuera el abogado estadounidense Luis Kutner, un activista de los derechos humanos y cofundador de Amnistía Internacional, quien en 1967 acuñó y difundió el término de Testamento Vital ${ }^{2}$ como "documento escrito en el que un ciudadano expresa los cuidados y tratamientos que querría tener en el caso de que por presentar una enfermedad importante u otro evento no estuviera consciente para una correcta comunicación". Paralelamente al Testamento Vital, en pleno desarrollo de la bioética, comenzaron a surgir los conceptos de limitación del esfuerzo terapéutico y muerte digna. Kutner defendió su testamento como una garantía de los derechos de los pacientes en la toma de decisiones. Un largo camino separa aquel enunciado del Testamento Vital en 1967 y la definición de Planificación de Decisiones Anticipadas (PDA) que la EAPC publicó por primera vez en $2017^{3}$.

La necesidad de esa participación del paciente en la toma de decisiones se fue consolidando académicamente en las siguientes décadas. Sin embargo, el estudio SUPPORT ${ }^{4}$, cuyos resultados se empezaron a publicar en 1995, ofreció evidencias rotundas respecto a la escasa atención que los clínicos prestaban a los deseos y a la trayectoria biográfica de los pacientes con enfermedades avanzadas. Los legisladores estimaron entonces necesaria la creación de leyes en prácticamente todos los países occidentales que facilitaran la expresión de esas decisiones por parte de los pacientes y garantizaran que eran tenidas en cuenta por los médicos. Entraron en escena los Documentos de Voluntades Anticipadas o de Instrucciones Previas, que supusieron un avance en el enunciado de los derechos de los pacientes pero en la práctica situaron la realización de esos documentos fuera del ámbito clínico.

En ese contexto surge la PDA como un instrumento clínico donde el equipo asistencial comparte con el paciente las distintas perspectivas de su situación clínica de cara a tomar decisiones para el futuro. Un intento de volver a llevar al ámbito clínico algo que es imprescindible e inherente a una medicina centrada en el paciente ${ }^{5}$. De ahí la editorial del New England tratando de cambiar el foco desde el instrumento legal al clínico ${ }^{6}$. Sin embargo, la PDA carecía de una definición concisa, se utilizaba en unos escenarios clínicos muy amplios con diversos objetivos y no existía una metodología definida exigible a cualquier instrumento clínico. Demasiadas cuestiones pendientes.

En este número se publica en español la definición de consenso de la PDA realizada por la EAPC a partir de un grupo de expertos internacionales con experiencia clínica y en investigación en el campo de la oncología, la atención paliativa, geriátrica y en ética. El grupo realizó un primer constructo de la definición y diseñó un extenso estudio Delphi que incluyó finalmente a un total de 124 profesionales sanitarios de 17 países que llegaron a un enunciado con un amplio grado de acuerdo. La PDA fue así definida como "la capacidad de permitir a las personas atendidas definir objetivos y preferencias sobre tratamientos y atención futuros, discutir esos objetivos y preferencias con los familiares y los profesionales responsables de la atención, y registrar y revisar esas preferencias cuando proceda"7.

La existencia de una definición ampliamente consensuada supone un indudable punto de inflexión para la investigación de la PDA como instrumento clínico. Quedan, sin embargo, muchas cuestiones pendientes. Los propios autores en el artículo hacen referencia a la inexistencia de un término consensuado en español para el Advance Care Planing. A pesar de utilizar en su artículo el término PDA, acuñado por el modelo catalán en 2015, creen más apropiada la traducción Planificación

1134-248X/ @ 2019 Sociedad Española de Cuidados Paliativos. Publicado por Inspira Network. Todos los derechos reservados. 
Compartida de la Atención (PCA) consensuada por el Grupo Español de PCA en 2017. Otros autores se han centrado más en el periodo final de la vida y hablan de Planificación Anticipada de la Atención al Final de la Vida o Planificación Anticipada de Cuidados. La diversidad en la terminología y los acrónimos en torno a un concepto que goza de un claro consenso no debe distraernos a la hora de responder a los interrogantes fundamentales y operativos: ¿quién, cuándo y cómo se debe realizar la PDA?, ¿cómo se integra la PDA en la práctica clínica?, ¿su uso permite mejorar la atención a los enfermos al final de la vida? Empezamos a tener respuestas para algunos de estos interrogantes.

El proceso de PDA incluye un uso general y otro específico. La PDA se puede utilizar en la población general sana o con problemas menores de salud, en lo que podemos denominar abordaje de tipo poblacional, que sería característico, aunque no exclusivo, de la Atención Primaria. Por otro lado, se puede hacer una utilización específica ante un diagnóstico de enfermedad progresiva e incurable, en lo que significa un abordaje en pacientes con patologías avanzadas. ¿Se deben considerar ambos procesos de la misma manera? Las perspectivas poblacionales enfatizan en la necesidad de que toda la población se conciencie de la necesidad de pensar en la etapa final de vida y determinar las preferencias genéricas sobre la atención. El ejemplo más documentado de esta perspectiva es el programa "Respecting Choices" que ha demostrado ya su utilidad en la práctica clínica ${ }^{8}$. En España, las experiencias de implementación de la PDA en el sistema asistencial catalán y vasco entrarían también dentro de esta perspectiva poblacional ${ }^{9}$. En todos estos programas la responsabilidad principal de la PDA recae en la Atención Primaria, aunque todos los profesionales deberían estar implicados en su realización. El abordaje en pacientes con patologías avanzadas requiere utilizar modelos que se enfocan específicamente al final de la vida con una metodología que responda a las necesidades de toma de decisiones en cada enfermedad. Las estrategias de desarrollo para la implementación de estos modelos recaen de manera preferente en los profesionales de cuidados paliativos aunque, en cualquier ámbito especializado, todos los profesionales deberían ser corresponsables de su realización. El programa LagunAdvance $^{10}$ sería un buen ejemplo en nuestro país de esta perspectiva centrada en fases avanzadas de la enfermedad (cáncer, insuficiencias orgánicas y enfermedades neurodegenerativas). El modelo parte de una concepción metodológica global por escalones para, a partir de ahí, desarrollar hojas de ruta específicas para cada patología en función de las decisiones que hay que tomar en las diferentes trayectorias de enfermedad. Ambos modelos son complementarios y sinérgicos.

Los estudios que existen en la práctica clínica empiezan a demostrar la eficacia de la PDA, desde en sus intervenciones más sencillas hasta en las más complejas, existiendo, además, un gradiente de efectividad, de modo que parece que cuanto más completa es la intervención, mejores resultados se obtienen en la práctica clínica ${ }^{11}$. Por ello, no resulta extraño que algunas sociedades científicas se hayan lanzado a publicar guías clínicas para la implementación de la PDA en los pacientes con enfermedades avanzadas ${ }^{12}$.

La PDA es un instrumento clínico básico en la atención de enfermos en estadios avanzados de enfermedad hasta el punto de que debiera estar presente en cualquier historia clínica. Los profesionales de cuidados paliativos deben, por tanto, conocer y familiarizarse con estos modelos, aprender una metodología concreta y prepararse para difundirla a otros profesionales. Estamos ante un área de investigación prioritaria que requiere invertir esfuerzos con urgencia. En los próximos años debemos ser capaces de definir las metodologías más apropiadas para elaborar la PDA y los mejores sistemas de aprendizaje e implementación en la práctica clínica. Ciertamente, necesitamos una revolución educativa13. Solo así podremos estandarizar este instrumento clínico, generalizar su utilización en las historias clínicas y facilitar su aprendizaje pre y postgrado. Tenemos un desafío que pondrá a prueba la calidad asistencial de los cuidados paliativos.

Alberto Alonso Babarro ${ }^{1}$ y Rogelio Altisent ${ }^{2}$

${ }^{1}$ Unidad de Cuidados Paliativos. Hospital Universitario La Paz. Madrid. ${ }^{2}$ Cátedra de Profesionalismo y Ética Clínica. Universidad de Zaragoza. IIS Aragón.

\section{Bibliografía}

1. Sulmasy DP, Brick C, Mackowiak PA. Eleanor Roosevelt's last days: a bioethical case study. Am J Med. 2015;128:437-40.

2. Simón Lorda P, Barrio Cantalejo IM. Un marco histórico para una nueva disciplina: la bioética. En: Couceiro Vidal A. Bioética para Clínicos. 1. a ed. Madrid: Ed Triacastela; 1999.

3. Rietjens JAC, Sudore RL, Connolly M, van Delden JJ, Drickamer MA, Droger M, et al. Definition and recommendations for advance care planning: an international consensus supported by the European Association for Palliative Care. Lancet Oncol. 2017;18:e543-e551.

4. Connors AF, Dawson NV, Desbiens NA, Fulkerson WJ, Goldman L, Knaus WA, et al. A controlled trial to improve care for seriously ill hospitalized patients. The study to understand prognoses and preferences for outcomes and risks of treatments (SUPPORT). JAMA. 1995; 274:1591-8.

5. Bardes CL. Defining "patient-centered medicine". N Engl J Med. 2012;366:782-3.

6. Rolnick JA, Asch DA, Halpern SD. Delegalizing Advance Directives - Facilitating Advance Care Planning. N Engl J Med. 2017;376:2105-7.

7. Lasmarías Martínez C, Delgado Girón S, Rietjens JAC, Korfage IJ, Gómez-Batiste X. Definición y recomendaciones para la Planificación de Decisiones Anticipadas: un consenso internacional apoyado por la European Association for Palliative Care (EAPC). Med Paliat. 2019;26:236-48. 
8. MacKenzie MA, Smith-Howell E, Bomba PA, Meghani SH. Respecting Choices and Related Models of Advance Care Planning: A Systematic Review of Published Evidence. Am J Hosp Palliat Care. 2018;35:897-907.

9. Lasmarías C, Aradilla-Herrero A, Santaeugènia S, Blay C, Delgado S, Ela S, et al. Development and implementation of an advance care planning program in Catalonia, Spain. Pall and Supp Care. 2019;17:415-24.

10. Villavicencio-Chavez C, Alonso-Babarro A, Rocafort J, Muñoz-Sánchez D, Gainza-Miranda D, Sanz-Peces EM, et al. LagunAdvance Planification Steps (LAP-S). Advance Care Planning in Patients with advanced disease. Individualizing the approach: chronic obstructive pulmonary disease (COPD), Amyotrophic lateral sclerosis (ALS), Dementia and Cancer. 10 ${ }^{\text {th }}$ World Research Congress of the European Association for Palliative Care. Bern, Switzerland. Palliat Med. 2018;32:278-9.

11. Brinkman-Stoppelenburg A, Rietjens JA, van der Heide A. The effects of advance care planning on end-of-life care: a systematic review. Palliat Med. 2014;28:1000-25.

12. Schrijvers D, Cherny NI; ESMO Guidelines Working Group. ESMO Clinical Practice Guidelines on palliative care: advanced care planning. Ann Oncol. 2014;25: iii138-42.

13. Altisent R. Planificación anticipada de la asistencia. Se necesita una revolución educativa. Aten Primaria. 2013;45:402-3. 